Conferencia Magistral

\title{
LOS NUEVOS PARADIGMAS DE LAS DEFENSORÍAS DEL PUEBLO. HOMENAJE AL Dr HÉCTOR GROS ESPIELL*
}

\author{
NEW PARADIGMS IN OMBUDSMAN'S OFFICES A. \\ TRIBUTE TO HÉCTOR GROS ESPIELL
}

\section{OS NOVOS PARADIGMAS DAS DEFENSORIAS DO POVO. HOMENAGEM AO Dr. HÉCTOR GROS ESPIELL}

Raúl Lamberto **

En este Seminario en homenaje a Héctor Gros Espiell, quien fuera uno de los cofundadores y presidente del ILO, intentaremos hacer un breve recorrido por el devenir de la institución del Defensor del Pueblo para concentrarnos especialmente en los nuevos aradigmas que marcarán su futuro.

Fueron los hombres de la ilustración (Rousseau, Montesquieu) los que llevarían a sostener la reducción del espacio de lo político como esfera de decisión del ejecutivo. A partir de los revolucionarios franceses de fines del siglo XVIII y comienzos del siglo XIX, se pueden investigar las condiciones históricas de la organización de la supremacía del poder legislativo y la desestimación del ejecutivo. La voluntad general es la obra del legislador, el despotismo se asimilaba al poder la particularidad. Uno de los grandes juristas franceses del siglo XX diría "legislar es un placer de una calidad más exquisita que mandar". Pierre Rosanvallon, en el libro "El buen gobierno", analiza la democracia-gobierno. Al término de la Primera Guerra Mundial, el fortalecimiento del ejecutivo se había producido en toda Europa bajo la presión de las necesidades; pero terminada la Segunda Guerra Mundial el espectro del nazismo y del fascismo, llevó a rechazar todo lo que pudiera emparentarse con una forma de personalización del poder. Se produjo entonces en Europa (aún con críticas y matices) un retorno al parlamentarismo .

El presidencialismo norteamericano, no parecía un sistema universalizable y se explicaba por las condiciones inaugurales de su independencia; sin embargo se constituiría en la

\footnotetext{
*Conferencia Magistral dictada en el marco del Seminario Regional sobre el "Rol de las Defensorías del Pueblo en la nueva agenda de los Derechos Humanos y sus desafíos regionales en el siglo XXI", llevado a cabo en la Facultad de Derecho UDELAR el 6 de diciembre 2019 en el marco del X homenaje a su fallecimiento. El Seminario fue organizado por ILO y contó con el apoyo de la INDDHH y el auspicio de la Defensoría de las Vecinas y Vecinos de MVD; la Facultad de Derecho de la UDELAR. En el marco de dicho Seminario fue instalado el CAPÍTULO URUGUAYO del Instituto Latinoamericano del ILO, en Asamblea realizada en el Palacio Legislativo el mismo día 6 de diciembre de 2020.
}

** Defensor del Pueblo de la Provincia de Santa Fe, Argentina. Correo electrónico: raullamberto@yahoo.com.ar 
gran alternativa democrática al parlamentarismo y fue el sistema adoptado por todos los países del hemisferio.

La Defensoría del Pueblo surge en el marco de la evolución de los sistemas políticos democráticos, especialmente en su vertiente parlamentaria, aunque luego alcanza también a la vertiente presidencialista. Esta idea de la preeminencia del cuerpo legislativo constituye la primera identificación de las Defensorías del Pueblo desarrolladas en la segunda mitad del siglo XX. Originalmente aparece bajo la monarquía en Suecia en 1809 mediante la figura del "Canciller de Justicia" o "justitieombudsman", cuya finalidad era supervisar la actuación de los funcionarios de la administración del reino ; en Inglaterra apareció bajo el nombre de "Comisario Parlamentario para la Administración" en 1967, con las particularidades británicas y en Francia como el "Mediateur de la République" . En la Península Ibérica cobra importancia la figura del "Diputado del Común” de Canarias, heredera de una tradición de defensa de las libertades públicas de los Procuradores del Común y de los Personeros de los siglos XVI a XVII, y que aparece como el vínculo con la figura del Defensor del Pueblo ibérico que conocemos desde el siglo XX.

Tras largas dictaduras que avasallaron los derechos humanos y ciudadanos, la creación de la Defensoría del Pueblo en España en 1978 y la del Provedor de Justicia en Portugal en 1975 marcan un punto de inflexión: además del control de la administración pública, estas nuevas instituciones asumen una clara función de protección de los derechos fundamentales, bajo un organismo de raigambre legislativa. El organismo creado dentro de la esfera legislativa fortalecía a ese poder frente a ejecutivos que se habían caracterizado por sus extralimitaciones; Latinoamérica, con su pasado de dictaduras, adoptó precisamente éste modelo de Defensoría del Pueblo.

La década de 1980 fue la de la caída de las dictaduras latinoamericanas y la del inicio de una era democrática en la que fueron discutidas nuevas instituciones e ideas tendientes a fortalecer el sistema democrático. En ese momento fundacional para nuestras democracias, Héctor Gros Espiell fue pionero al exponer las razones, el sentido y la oportunidad para que hubiese un ombudsman en Latinoamérica. Quizás uno de los mayores méritos de Gros Espiell haya sido advertir que por sus características cultural y socialmente complejas, Latinoamérica debía dotarse de una institución del ombudsman distinta y superadora de los modelos ya existentes.

En septiembre de 2017, el politólogo francés Alain Rouquié -experto en historia latinoamericana y argentina- visitó la provincia de Santa Fe y solicitó especialmente conocer la Defensoría del Pueblo, le interesaba conocer en profundidad los resortes de la institución en su variante latinoamericana. Rouquié se mostró particularmente interesado por la misión de protección de derechos que tiene la Defensoría del Pueblo, tal como se la concibe en Latinoamérica. Fue harto elogioso con el organismo, del cual subrayó su "tarea, fortaleza y credibilidad". 
Durante el encuentro, Rouquié expresó que en el paradigma de los sistemas políticos presidencialistas -el estadounidense- existe una voluntad transgresora por parte del poder ejecutivo, pero que es claramente limitada por el Capitolio. La existencia de un poder legislativo fuerte es lo que pone un coto a las ambiciones de un ejecutivo poderoso creado bajo el modelo de "un rey que muere cada cuatro años". Sin embargo, en su emulación del presidencialismo estadounidense, los países de Latinoamérica sobredimensionaron aún más la fortaleza del ejecutivo ante un poder legislativo débil.

La Defensoría del Pueblo en su variante latinoamericana, tal como señala Carlos Constenla, dejó de ser un organismo de control de la administración para convertirse en un organismo de protección de derechos.

Nuestras sociedades se han tornado cada vez más complejas y desafían a las instituciones tradicionales. Asumir el "paradigma de la complejidad" resulta indispensable para que instituciones como las Defensorías del Pueblo puedan ofrecer respuestas a las sociedades actuales y a sus reclamos en permanente evolución.

Los Derechos Económicos, Sociales y Culturales irrumpen en la agenda defensorial y exigen un nuevo compromiso en la evaluación y el monitoreo de implementación de políticas públicas por parte del Estado. Las Defensorías del Pueblo no pueden ya desentenderse del derecho al trabajo, que se impone como un reclamo cada vez más potente. Tampoco del derecho a un ambiente sano y sostenible. Asimismo, las Defensorías del Pueblo tienen el deber de replantearse una mayor cercanía con la ciudadanía y la ampliación de su alcance territorial, en atención principalmente a las necesidades de los sectores más vulnerables. Como dijimos, las Defensorías de órganos de control deben convertirse en organismo de protección de derechos.

Las modernas Defensorías no pueden resignar las funciones de control y de defensa del ciudadano frente a los actos, hechos y omisiones de la Administración Pública que impliquen un ejercicio ilegítimo, abusivo, arbitrario, discriminatorio, negligente, gravemente inconveniente de sus funciones, o configuren una desviación de poder. Sin embargo, la realidad actual origina nuevos desafíos. Un primer objetivo es extender la visibilidad de las Defensorías, su alcance territorial y la incorporación de tecnología que permita una comunicación fluida y una capacidad de respuesta a la ciudadanía acorde a las dinámicas actuales.

Mayor alcance territorial para reducir las desigualdades La sociedad multiplica sus reclamos pero no siempre puede expresarlos dónde y cómo debiera para encontrar respuestas. En otras palabras, las Defensorías del Pueblo hoy tienen el desafío de ir en busca de esos reclamos y ampliar el alcance territorial. En tal sentido, la creación de oficinas móviles implementada por la Defensoría del Pueblo de la provincia de Santa Fe, puede ser considerada como una experiencia importante en tanto constituye 
una buena práctica tendiente a la reducción de las desigualdades.

Para comprender el verdadero significado y la importancia de este proyecto, hay que destacar que la Defensoría del Pueblo de Santa Fe es un organismo con competencia en todo el territorio de la provincia, la que se caracteriza por su extensión territorial de más de 720 kilómetros de longitud de norte a sur y de 380 kilómetros en su eje este-oeste, con 55 municipios y 308 comunas.

El programa de oficinas móviles tuvo por objetivo principal convertir a la Defensoría en un organismo de proximidad, presente en el territorio para atender la demanda de toda la población, principalmente la más vulnerable.

El proyecto se implementó en el mes de junio del 2018, y para su puesta en marcha se dispuso de dos vehículos tipo utilitario, adaptados en su interior para la atención de los ciudadanos con el equipamiento informático y conectividad necesaria para brindar la atención en territorio.

El programa Oficina Móvil atendió a 3575 casos entre junio de 2018 y noviembre de 2019, se realizaron 151 visitas a localidades del interior provincial y 55 visitas a barrios de las ciudades de Santa Fe y Rosario. Más del 60 por ciento de los reclamos fue resuelto con la atención, requiriendo los restantes un trámite posterior en las delegaciones o en las sedes.

Considerando las actividades desplegadas, la cantidad de reclamos atendidos, la recepción y apertura de las localidades visitadas, se puede concluir que el dispositivo de las oficinas Oficinas Móviles es una herramienta novedosa y adecuada para dar respuesta una necesidad de mayor alcance territorial. Joseph Stiglitz (premio Nobel de economía) sostiene que las sociedades demasiado desiguales son menos eficaces y sus economías no son estables ni sostenibles, las desigualdades económicas llevan indefectiblemente a las desigualdades políticas. Martin Luther King expresaba que las injusticias del pasado no eran inevitables, pero sabía también que no bastaba con soñar. Las Defensoría del Pueblo no deben soñar sino trabajar. En el informe sobre políticas sociales en la ciudad de Rosario, uno de los temas abordados fue el de la pobreza, que socava los valores de la democracia y pone en entredicho su eficacia.

Las políticas públicas con enfoque de derechos constituyen obligaciones de la institucionalidad pública del Estado de garantizar progresivamente mayores niveles de igualdad a todas las personas, sin discriminación de ningún tipo.

El derecho a la ciudad ha sido definido como el derecho de toda persona a vivir dignamente en un espacio público colectivo, con un gobierno elegido democráticamente, que tenga como centro de sus políticas públicas a las personas y el respeto a sus derechos humanos.

El derecho a la ciudad implica el reconocimiento de la ciudad como un bien colectivo, 
como derecho humano colectivo, resultado de una lucha sistemática de los movimientos sociales que reúne múltiples dimensiones de la vida común en un espacio potencial de igualdad. Es un derecho que exige instrumentos de gestión participativos para tornarlo efectivo. Se trata de un derecho a consagrar como tal, y se trata en última instancia, de hacer visibles y vivibles las ciudades invisibles a las que se refería Italo Calvino En los últimos decenios, el mundo ha experimentado un crecimiento urbano sin precedentes: la mitad de la humanidad, esto es unos 3.500 millones de personas, vive actualmente en ciudades y esta cifra seguirá aumentado. El futuro será, sin dudas, urbano para la mayoría de las personas. Por lo tanto, las soluciones a algunos de los principales problemas a los que se enfrentan (pobreza, cambio climático, educación, trabajo, salud pública, etc.) deben encontrarse en el marco de la ciudad.

La rápida urbanización conlleva enormes dificultades vinculadas a la integración y a la calidad de vida de las personas, tales como: creciente número de habitantes de barrios marginales; incremento de la contaminación atmosférica; insuficiencia de servicios públicos básicos e infraestructura de viviendas; crecimiento urbano incontrolado y no planificado; aumento de las vulnerabilidades en las condiciones de vida y de los desastres. El número absoluto de residentes en zonas urbanas, que viven en barrios marginales sigue aumentando, en parte debido a la aceleración de la urbanización, el crecimiento demográfico y la falta de políticas apropiadas en materia de tierras y viviendas.

En la reunión de Hábitat III (Quito 2016), las Defensorías del Pueblo de Latinoamérica asumieron ante ONU el compromiso de monitorear, en sus respectivos países, el avance de medidas y dispositivos adoptados en el nivel nacional y subnacional, destinados a cumplir con los objetivos de la Nueva Agenda Urbana, que se se complementa con la Agenda 2030 y sus Objetivos de Desarrollo Sostenible (2015) .

Esta función de monitoreo constituye un aspecto relevante e innovador dentro del amplio espectro de las funciones de las Defensorías .

El vínculo entre la Defensoría del Pueblo y la Academia

La Defensoría celebró un convenio con la Universidad Nacional de Rosario cuyo objeto fue reunir un grupo de investigadores especializados, bajo la coordinación de dos académicos de amplia trayectoria.

En el año 2017 el estudio y análisis se circunscribió territorialmente a la ciudad de Rosario. Se investigó sobre las políticas públicas de los poderes ejecutivos nacional provincial y local, en torno a pobreza, vejez, discapacidad, violencia de género, servicios y vivienda. En el año 2018, la estrategia de trabajo asumió tres nuevos desafíos: 1- aumentar la escala y evaluar todo el territorio de la provincia; 2- centrar la evaluación en los derechos económicos, sociales y culturales (DESC) y no principalmente en las políticas sociales y 3- evaluar al los tres poderes Judicial, Legislativo y Ejecutivo. 
El objetivo del Equipo fue evaluar el estado de avance de los derechos a la salud, a la educación, a la seguridad social, a una vivienda adecuada y servicios públicos básicos y a una vida libre de violencia en la Provincia de Santa Fe, considerando en particular a las personas con discapacidad, a los adultos mayores, a la población en condición de pobreza y atravesada por las desigualdades de género .

En el trabajo se consideró especialmente el análisis del contexto financiero y el compromiso presupuestario en torno a cada uno de los derechos monitoreados, en base a las fuentes de financiamiento y a la evaluación del gasto público en la materia. El libro de Stephen Holmes y Cass R. Sunstein de la editorial Siglo XXI, titulado "El costo de los derechos" es una buena introducción a un tema ignorado y que se decidió evaluar especialmente en la investigación de la Defensoría y la UNR.

Además se analizó la información pública oficial y se construyó una matriz de vacancia en el acceso a la información que intenta resignificar los vacíos y orientar al Estado en la producción de información pública en materia de derechos humanos.

Se produjo un Informe final de evaluación basado en los hallazgos sobre el progreso de los cinco derechos económicos, sociales y culturales seleccionados en la Provincia de Santa Fe, y se formularon recomendaciones para la acción pública. Se identificaron avances progresivos en los cinco derechos humanos evaluados. Se ponderaron avances y se señalar caminos posibles de mejoramiento progresivo.

El mundo ha cambiado: la libre circulación de mercancías, la globalización, la revolución informática, la eliminación de fronteras del comercio ha causado transformaciones radicales.

Las normas laborales sólo pueden ser admitidas en la medida en que no obstaculicen la realización de este objetivo. El Acuerdo de Marrakech, instituye la Organización Mundial del Comercio (OMC) con lo cual la competición global se postula bajo la premisa de que cada país debía explotar su ventaja comparativa. Indicadores económicos cuantificables (tasa de empleo, volumen de ingresos, demanda efectiva, incremento de la producción y el comercio) son tratados como fines en sí mismos. Los seres humanos han desaparecido de la lista de objetivos asignados a la economía y al comercio. Así se instala la competencia por el menor coste social, laboral o medioambiental.

Esta inversión de medios y fines solo lleva a la catástrofe, porque, un ordenamiento jurídico que sitúa las cosas por encima de los hombres no puede ser viable.

Pensar el futuro del trabajo es fundamental para un desarrollo sostenible que ponga fin a la pobreza y "no deje a nadie atrás". Ese futuro del trabajo debe plantearse en torno a dos ejes centrales:

1) debe estar centrado en las personas,

2) debe proponerse alcanzar la justicia social. 
Al establecer estos dos ejes, lo que se pretende es poner los derechos de los trabajadores y las necesidades, las aspiraciones y los derechos de todas las personas en el núcleo de las políticas económicas, sociales y ambientales.

Es necesario mejorar la calidad de vida de los trabajadores, ampliar las opciones disponibles, cerrar la brecha de género, revertir los estragos causados por las desigualdades a nivel mundial.

Las Defensorías del Pueblo tienen mucho que decir respecto de la orientación, la formación y la sensibilización respecto de la violencia y acoso en el mundo del trabajo.

El enfoque novedoso debe estar en el reconocimiento de las personas y no en la distribución de bienes, el centro debe ser el trabajo en sí, en lo que la OIT llama un trabajo realmente humano.

La Defensoría del Pueblo de la provincia de Santa Fe, por su trayectoria en el abordaje de la violencia laboral, integró la Delegación Oficial Argentina en la $108^{\circ}$ Conferencia de la OIT en junio de 2019, donde se aprobó el Convenio sobre Violencia y Acoso Laboral y se establecieron las bases sobre el futuro del trabajo.

La cuestión ambiental plantea grandes desafíos que deben ser asumidos con la mayor seriedad y atención por las Defensorías del Pueblo.

Se debe trabajar para apuntalar el concepto de desarrollo sostenible, definido como "la satisfacción de las necesidades de la generación presente sin comprometer la capacidad de las generaciones futuras para satisfacer sus propias necesidades".

Los problemas ambientales son reales y requieren acciones concretas y urgentes

El Cambio Climático es el mal de nuestro tiempo, sus consecuencias pueden ser desbastadoras si no se reducen las emisiones de gases de efecto invernadero (GEI). En 2016 el aumento global de temperatura fue de 1.1 grados centígrados .

Debe fomentarse el uso de energías más eficientes, de baja o nula emisión de GEI; promover proyectos de prosumidores y generación de energías alternativas con incentivos desde el estado; difundir y concienciar sobre la problemática.

La deforestación es un drama mundial que nos toca muy de cerca en América Latina. Cada año desaparecen 10 millones de hectáreas de bosques primarios, de seguir a este ritmo, en menos de 300 años se acabaran los árboles del planeta. La FIO se pronunció expresamente sobre la catástrofe de la Amazonia.

Debe fomentarse la reforestación y la disminución en el consumo de maderas.

Los humedales del mundo están desapareciendo a un ritmo tres veces mayor que los bosques. Los humedales son vitales para la supervivencia humana.

Es importante también atender las cuestiones vinculadas a la Agricultura Familiar, Soberanía y Seguridad Alimentaria, como así, al tratamiento de residuos.

En palabras del ex Secretario General de la ONU, Ban Ki moon "El cambio climático es 
la causa más importante de nuestro tiempo".

Las Defensorías del Pueblo enfrentan un cambio de paradigma producto de los cambios sociales y de la ampliación de derechos que las sociedades modernas exigen. Es por esos motivos que, tratándose de la institución más joven de la democracia, se espera de ella una mayor versatilidad al momento de adaptarse a los cambios para ofrecer respuesta sin perder su identidad.

Como conclusión general y a modo de sugerencia, es importante destacar algunos puntos a tener en cuenta como tendencias en los nuevos paradigmas de las Defensorías del Pueblo:

- Los Estados y las Defensorías del Pueblo como organismos protectores de derechos enfrentan realidades sociales y culturales cada vez más complejas y deben prepararse para ofrecer respuestas en ese sentido.

- $\quad$ Es necesario avanzar en la institucionalización del Enfoque de Derechos en el Estado.

- $\quad$ Ningún Estado, en ningunos de sus tres niveles, parte desde cero.

- Las Defensorías del Pueblo NO implementan políticas públicas. Su función está justamente ligada a la evaluación y el monitoreo de las políticas públicas.

- Deben generarse vínculos estratégicos entre distintos organismos para poder evaluar y monitorear políticas sociales. En este sentido, la alianza estratégica de las Defensoría del Pueblo con la Academia aparece como una experiencia a tener en cuenta.

- El compromiso presupuestario es una condición para el cumplimiento de los derechos y tanto las fuentes de financiamiento como la evolución del gasto público deben estar comprendidos en la evaluación de las políticas sociales.

- $\quad$ El derecho a la información es también un presupuesto necesario para el seguimiento y evaluación de la implementación de políticas sociales.

- $\quad$ Es función esencial de las Defensorías del Pueblo, velar por los derechos de las personas, priorizando la protección de los más vulnerables, en pos de la justicia social, basada en el concepto de trabajo centrado en la persona y no en la variable económica asociada y con lineamientos de protección de la naturaleza como proveedora principal de los bienes.

- $\quad$ Es importante trabajar para remover los obstáculos que dificultan a distintos sectores de la población el acceso a las Defensorías, para lo cual las oficinas móviles resultan una herramienta a tener en cuenta.

- Defender a las Defensorías. La institución del Ombudsman se encuentra bajo amenaza en distintas partes del mundo y en nuestra región. Recientemente la FIO se pronunció sobre la preocupante situación en Ecuador, Guatemala, Chile y Bolivia. En los próximos días se llevará adelante en Cartagena de Indias, Colombia, el Foro "Defender 
a los Defensores: 71 años de la Declaración Universal de los Derechos Humanos", con el objetivo de generar un espacio de reflexión y análisis en torno al rol que cumplen los defensores de derechos humanos en la sociedad y cómo se debe dar un esfuerzo interinstitucional por conocerlos y garantizarles las condiciones adecuadas para el ejercicio de su labor.

La Defensoría del Pueblo de la provincia de Santa Fe, en virtud de leyes sancionadas por la legislatura provincial, tiene en pleno funcionamiento la Defensoría de niñas, niños y adolescentes; es autoridad de aplicación de la ley de salud mental; tiene sancionada la norma y está en proceso de implementación la Defensoría de la tercera edad y el Observatorio de políticas en materia de discapacidad. Es decir que tenemos la responsabilidad institucional de abordar los temas que constituyen los objetivos y metas de la agenda 2030. Nuestra tarea es ardua y requiere una gran responsabilidad. En ese camino estamos transitando la línea de desarrollo propuesta por Héctor Gros Espiell, que marca el futuro de las Defensorías del Pueblo de nuestra querida Latinoamérica.

Montevideo, 6 de diciembre 2019 